\title{
Totally extraperitoneal approach to laparoscopic lateral lymph node dissection for patients with recurrent lateral pelvic lymph nodes after rectal cancer surgery: a novel technique-M TEP LLND
}

\author{
Shinsuke Masubuchi ${ }^{1}$ - Junji Okuda ${ }^{2} \cdot$ Hiroki Hamamoto $^{1} \cdot$ Masatsugu Ishii $^{1}$ ' Wataru Osumi ${ }^{1}$. \\ Masashi Yamamoto ${ }^{1} \cdot$ Yoshihiro Inoue $^{1} \cdot$ Keitaro Tanaka $^{1} \cdot$ Kazuhisa Uchiyama $^{1}$
}

Received: 14 January 2019 / Accepted: 31 March 2019 / Published online: 10 April 2019

(c) The Author(s) 2019

\begin{abstract}
Lateral lymph node dissection (LLND) for recurrence of lateral pelvic lymph node metastasis after rectal cancer surgery is technically demanding because of the need for re-do surgery. We herein report a novel technique of laparoscopic LLND via a totally extraperitoneal (TEP) approach. Since October 2018, we have performed LLND based on a TEP approach, called "M TEP LLND", with two cases treated. By peeling in the caudal direction in the dorsal layer of the rectus abdominis muscle, a working space is created once the extraperitoneal space is reached, and LLND is performed. All lateral pelvic lymph node dissection procedures have been successfully completed, and there have been no intraoperative or postoperative complications. This procedure allows TEP-experienced colorectal surgeons to perform safe and complete LLND without any influence of intraperitoneal adhesion or intestinal obstruction. M TEP LLND is less invasive than the conventional intraperitoneal approach and appears to be useful, particularly for recurrence of lateral pelvic lymph node metastasis.
\end{abstract}

Keywords Extraperitoneal approach $\cdot$ Lateral lymph node dissection $\cdot$ Totally extraperitoneal (TEP)

\section{Introduction}

Patients with local recurrence after rectal cancer surgery have a poor prognosis. Lateral pelvic lymph node metastasis is one of the major causes of local recurrence after total mesorectal excision (TME) [1, 2]. Local recurrence limited to lateral pelvic lymph nodes can be potentially treated by salvage lateral pelvic lymph node dissection (LLND). The safety and feasibility of laparoscopic LLND with TME for primary rectal cancer have been reported, but only a few studies have assessed laparoscopic salvage LLND for recurrent lateral pelvic lymph nodes [3-6]. LLND for recurrence of lateral pelvic lymph node metastases is technically demanding because of the need for re-do surgery.

Shinsuke Masubuchi

sur124@osaka-med.ac.jp

1 Department of General and Gastroenterological Surgery, Osaka Medical College, 2-7 Daigaku-machi, Takatsuki 569-8686, Japan

2 Cancer Center, Osaka Medical College, Takatsuki 569-8686, Japan
We herein report a novel technique via a totally extraperitoneal (TEP) approach to laparoscopic LLND for patients with recurrent lateral pelvic lymph nodes after rectal cancer surgery.

\section{Material and methods}

Since October 2018, we have been performing LLND with a TEP approach, called "M TEP LLND", for patients with resectable lateral lymph node recurrence after rectal cancer surgery; two cases have been experienced thus far.

Patients with local recurrence limited to lateral pelvic lymph nodes with the likelihood of an R0 resection are candidates for M TEP LLND. All patients were evaluated by abdominal computed tomography (CT) regularly during follow-up after the initial surgery. Patients who were found to have enlarged lateral pelvic lymph nodes underwent magnetic resonance imaging (MRI), and treatment was discussed at our board conference. Patient 1 was a 69 -year-old man who had undergone laparoscopic intersphincteric resection without LLND 7 months earlier. We diagnosed left lateral pelvic lymph node recurrence pathologically as one positive 
node. Patient 2 was a 73-year-old woman who had undergone laparoscopic low anterior resection without LLND 1 year and 8 months earlier. We diagnosed left lateral pelvic lymph node recurrence pathologically as one positive node (Table 1). The CT and MRI images are shown in Fig. 1.

\section{Surgical technique}

This procedure is based on the TEP method for inguinal hernia surgery [7]. It begins with a small incision (about

Table 1 Characteristics of patients

\begin{tabular}{lll}
\hline & Patient 1 & Patient 2 \\
\hline Sex & Male & Female \\
Age (years) & 69 & 73 \\
BMI & 31.6 & 21.5 \\
Primary operation & Laparoscopic & Laparo- \\
& intersphinc- & scopic \\
& teric resection & low \\
& & anterior \\
& & resection \\
Histology & Moderately & Well-differ- \\
& differentiated & entiated \\
& adenocarci- & adenocar- \\
& noma & cinoma \\
Pathological stage of primary cancer & Stage I & Stage II \\
Interval between primary surgery and & 7 & 20 \\
salvage surgery (months) & & \\
\hline
\end{tabular}

$B M I$ body mass index
$15 \mathrm{~mm}$ ) below the umbilicus. The incision of the anterior sheath of the rectus abdominis muscle is then made. Next, the rectus abdominis muscle is laid from the midline to the outside, and a 12-mm port is inserted in front of the posterior lamina of the rectus sheath. A camera is inserted through this port, and a $5-\mathrm{mm}$ port is inserted in the midline between the umbilicus and the pubis. Peeling toward the caudal direction in the dorsal layer of the rectus abdominis muscle is performed, and on reaching the extraperitoneal space, and a $5-\mathrm{mm}$ port is inserted $1 \mathrm{~cm}$ superior the median pubis (Fig. 2a, b).

First, with the pubic bone and Cooper's ligament as landmarks, the external iliac vein is exposed. On identifying the obturator foramen, the obturator nerve is preserved, but the obturator vessels are divided. The extent of lymph node dissection is along the obturator internus muscle at the outer edge and along the bladder at the inner edge. The distal side of the dissection line is at Alcock's canal. The branches of the internal iliac artery are clipped and divided (ex. superior vesical artery, inferior vesical artery, obturator artery, artery to the ductus deferens or uterine artery). The surface of the internal iliac vein is exposed, and the branches of the internal iliac vein are also clipped and divided. In cases in which the metastatic lateral pelvic lymph nodes have adhered or are close to the internal iliac artery, the artery is resected en bloc along with the lateral pelvic lymph nodes. The dissected lymph node is placed in the bag and removed from the 12-mm port wound (Fig. 2c, d).
Fig. 1 Representative abdominal computed tomography (CT) and magnetic resonance imaging (MRI) findings of patients 1 and 2. a Follow-up CT of patient 1 after primary surgery revealed left lateral pelvic lymph node recurrence. b Follow-up MRI of patient 1 after primary surgery revealed left lateral pelvic lymph node recurrence. c Follow-up CT of patient 2 after primary surgery revealed left lateral pelvic lymph node recurrence. d Follow-up MRI of patient 2 after primary surgery revealed left lateral pelvic lymph node recurrence
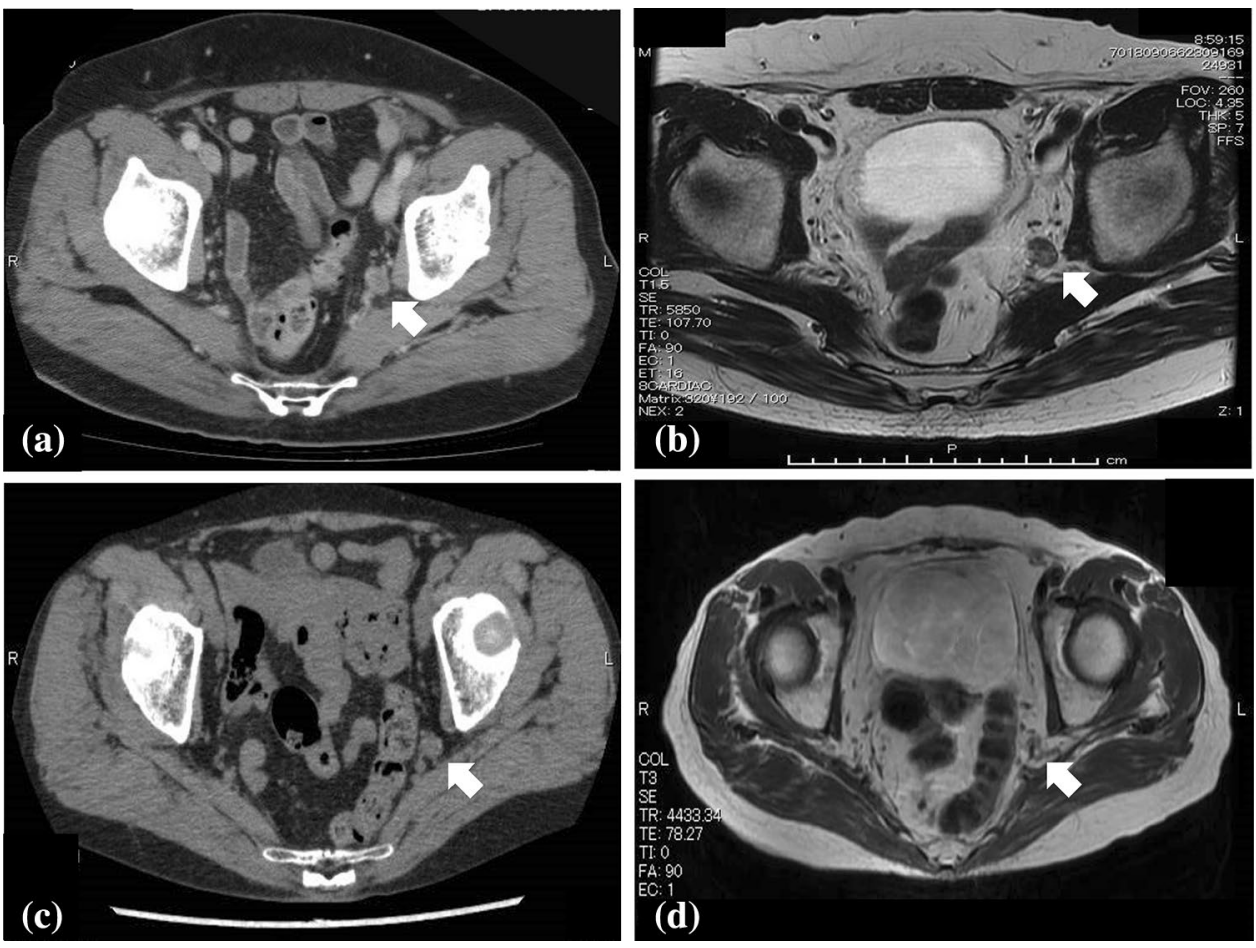
(a)
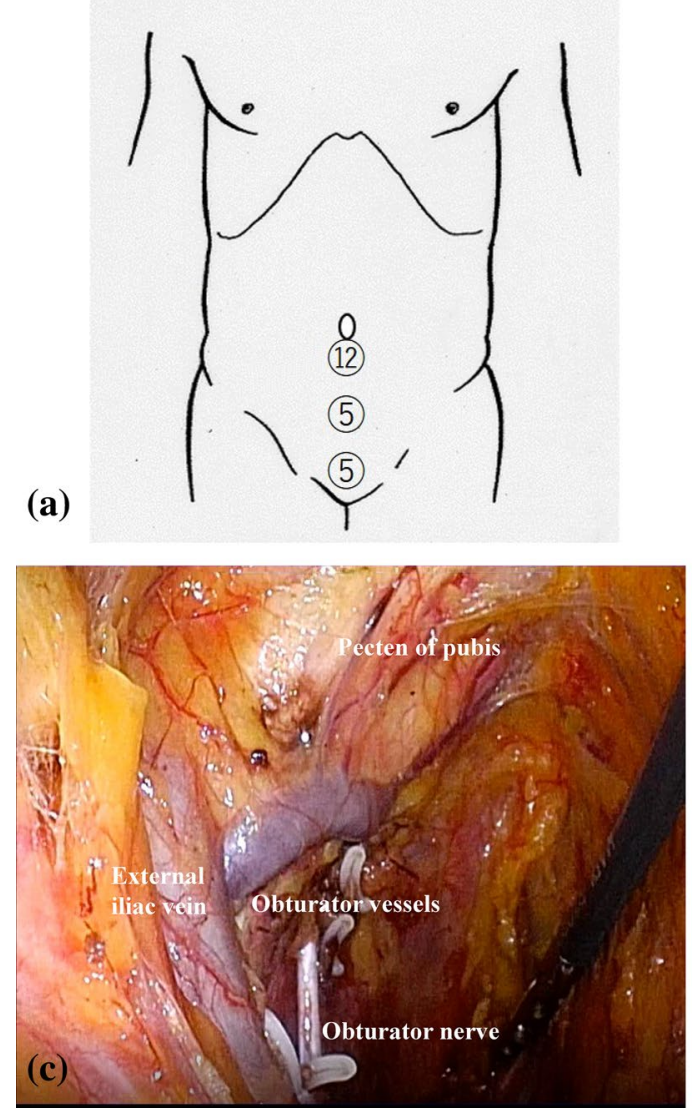
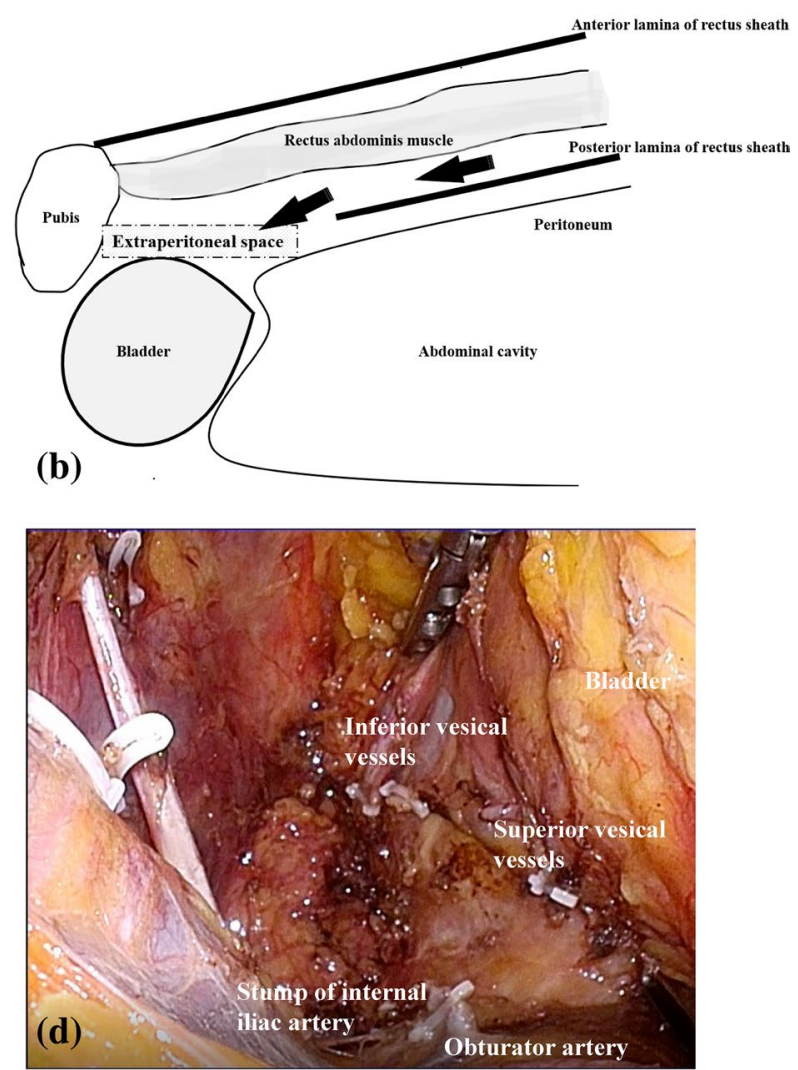

Fig. 2 a Port placement. b Approach to the extraperitoneal space. We peeled toward the caudal direction in the dorsal layer of the rectus abdominis muscle and reached the extraperitoneal space. c, $\mathbf{d}$ Laparoscopic view after lateral pelvic lymph node dissection

Table 2 Perioperative data

\begin{tabular}{lll}
\hline & Patient 1 & Patient 2 \\
\hline Operative time (min) & 231 & 243 \\
Blood loss (ml) & 10 & 10 \\
Adjacent structures removed en bloc & Internal iliac artery & None \\
Number of retrieved lymph nodes & 7 & 9 \\
Number of positive lymph nodes & 1 & 1 \\
Postoperative complication & None & None \\
Postoperative hospital stay (days) & 8 & 10 \\
\hline
\end{tabular}

\section{Results}

The perioperative data are summarized in Table 2. The operation time was $231 \mathrm{~min}$ for patient 1 and $243 \mathrm{~min}$ for patient 2 . The estimated blood loss was $10 \mathrm{ml}$ in both. The adjacent structures removed en bloc were only the internal iliac artery in patient 1 . R0 resection was achieved in both patients. The number of retrieved lymph nodes was seven for patient 1 and nine for patient 2 . In both, the number of metastatic lymph nodes was 1 . The postoperative length of hospital stay was 8 days for patient 1 and 10 days for patient 2 . There were no postoperative complications.

\section{Discussion}

Laparoscopic surgery provides a highly detailed image and magnifying effect, so it is extremely useful in cases of complicated surgery, such as salvage LLND performed in a very deep, narrow pelvic space. To our knowledge, few studies have focused on salvage surgery for recurrence of lateral pelvic lymph node metastasis after rectal cancer surgery [6].

Laparoscopic salvage LLND has been reported as a minimally invasive surgery because of its low blood loss $[6,8]$. However, the influence of re-operation must also be considered, as adhesion of the small intestine and reconstructed colon to the pelvis makes laparoscopic LLND more difficult. However, the extraperitoneal approach allows TEPexperienced colorectal surgeons to perform safe and complete LLND without any adverse effects of intraperitoneal adhesion and intestinal obstruction.

In the present cases, we were able to remove all of the lymph nodes around the internal iliac artery, external iliac 
artery, and obturator nerve. However, this technique has limited use when applied to dissection of lymph nodes around the common iliac artery. This technique can be performed as solo surgery without an assistant port. However, in our cases, surgery was performed with adequate staffing and the ability to transition to the intraperitoneal approach at any time to cope with unexpected intraoperative bleeding. There have been only a few reports of laparoscopic pelvic lymph node dissection via an extraperitoneal approach, so a thorough anatomical understanding is necessary for this approach $[9,10]$.

The technique reported this time is based on the TEP hernia repair method for inguinal hernia surgery, and we have termed it "M TEP LLND". M TEP LLND is less invasive than the conventional intraperitoneal approach and appears to be useful, particularly for recurrent lateral pelvic lymph node metastasis. In this report of this method, the number of cases was small, and the observation period was short. In the future, it will be necessary to assess the long-term oncological outcomes of this method.

\section{Conclusion}

The TEP approach to laparoscopic lateral lymph node dissection for patients with recurrent lateral pelvic lymph nodes after rectal cancer surgery is technically feasible and safe when performed by TEP-experienced colorectal surgeons. Although the short-term outcomes in the present cases were acceptable, the study was limited by its small size, and further investigations are needed to assess the oncological longterm outcomes of this technique.

Acknowledgements We thank Dr. Takashi Kinoshita (Hirakata City Hospital, Osaka, Japan) for his support in preparing the report.

\section{Compliance with ethical standards}

Conflict of interest Drs. Shinsuke Masubuchi, Junji Okuda, Hiroki Hamamoto, Masatsugu Ishii, Wataru Osumi, Masashi Yamamoto, Yoshihiro Inoue, Keitaro Tanaka and Kazuhisa Uchiyama have no conflicts of interest or financial ties to disclose.

Open Access This article is distributed under the terms of the Creative Commons Attribution 4.0 International License (http:// creativecommons.org/licenses/by/4.0/), which permits unrestricted use, distribution, and reproduction in any medium, provided you give appropriate credit to the original author(s) and the source, provide a link to the Creative Commons license, and indicate if changes were made.

\section{References}

1. Enriquez-Navascues JM, Borda N, Lizerazu A, Placer C, Elosegui JL, Ciria JP, et al. Patterns of local recurrence in rectal cancer after a multidisciplinary approach. World J Gastroenterol. 2011;17:1674-84.

2. Akiyoshi T, Ueno M, Matsueda K, Konishi T, Fujimoto Y, Nagayama $\mathrm{S}$, et al. Selective lateral pelvic lymph node dissection in patients with advanced low rectal cancer treated with preoperative chemoradiotherapy based on pretreatment imaging. Ann Surg Oncol. 2014;21:189-96.

3. Konishi T, Kuroyanagi H, Oya M, Ueno M, Fujimoto Y, Akiyoshi $\mathrm{T}$, et al. Multimedia article. Lateral lymph node dissection with preoperative chemoradiation for locally advanced lower rectal cancer through a laparoscopic approach. Surg Endosc. 2011:25:2358-9.

4. Liang JT. Technical feasibility of laparoscopic lateral pelvic lymph node dissection for patients with low rectal cancer after concurrent chemoradiation therapy. Ann Surg Oncol. 2011;18:153-9.

5. Park JS, Choi GS, Lim KH, Jang YS, Kim HJ, Park SY, et al. Laparoscopic extended lateral pelvic lymph node dissection following total mesorectal excision for advanced rectal cancer: initial clinical experience. Surg Endosc. 2011;25:3322-9.

6. Akiyoshi T, Nagata J, Nagasaki T, Konishi T, Fujimoto Y, Nagayama S, et al. Laparoscopic salvage lateral pelvic lymph node dissection for locally recurrent rectal cancer. Colorectal Dis. 2015; 17:213-6.

7. Wakasugi M, Masuzawa T, Tei M, Omori T, Ueshima S, Tori M, et al. Single-incision totally extraperitoneal inguinal hernia repair: our initial 100 cases and comparison with conventional three-port laparoscopic totally extraperitoneal inguinal hernia repair. Surg Today. 2015;45:606-10.

8. Yang TX, Morris DL, Chua TC. Pelvic exenteration for rectal cancer: a systematic review. Dis Colon Rectum. 2013;56:519-31.

9. Querleu D, Ferron G, Rafii A, Bouissou E, Delannes M, Mery E. Pelvic lymph node dissection via a lateral extraperitoneal approach: description of a technique. Gynecol Oncol. 2008;109:81-5.

10. Hsu T, Su LM, Ong A. Anterior extraperitoneal approach to laparoscopic retroperitoneal lymph node dissection: a novel technique. Urology. 2003;62:1114-6.

Publisher's Note Springer Nature remains neutral with regard to jurisdictional claims in published maps and institutional affiliations. 